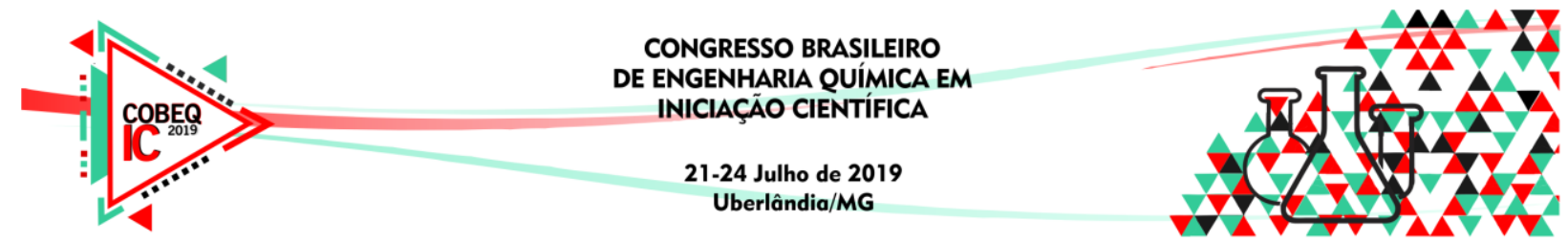

\title{
SÍNTESE, CARACTERIZAÇÃO E ATIVIDADE ELETROCATALÍTICA FRENTE RRO DE UM NOVO COMPLEXO DE NÍQUEL (II) CONTENDO LIGANTE DO TIPO N,O-DOADOR
}

\author{
M. M. DINIZ ${ }^{1}$ A. CASELLATO ${ }^{1}$, F. S. MOURA ${ }^{1}$, T. D. PETIZERO ${ }^{1}$, F. P. da \\ SILVA $^{2}$ \\ ${ }^{1}$ Universidade Federal Do Rio de Janeiro, Instituto de Química \\ ${ }^{2}$ Universidade Federal Do Rio de Janeiro, Escola de Química \\ E-mail para contato: dinizmmatheus@gmail.com
}

\begin{abstract}
RESUMO - As pilhas à combustível são uma opção promissora na geração de energia elétrica limpa. A platina é o principal eletrocatalisador utilizado nesses dispositivos. O elevado custo e baixa disponibilidade desse metal tornam necessário investigar novos compostos que possam desempenhar o papel da platina nessas pilhas. O objetivo do presente trabalho consiste justamente em sintetizar, caracterizar e avaliar a atividade de um novo composto de coordenação que possa eletrocatalisar as reações de redução do oxigênio em meio básico.
\end{abstract}

\section{INTRODUÇÃO}

A sociedade tem discutido cada vez mais sobre os problemas gerados pelo crescimento da dependência dos combustíveis fósseis como principal fonte de energia elétrica. As pilhas à combustível surgem, então, como uma alternativa promissora para gerar energia elétrica de forma ecológica e eficiente. Esses dispositivos utilizam a platina como principal eletrocatalisador de suas reações. A platina é um metal nobre, que possui alto custo e sua disponibilidade é reduzida, esses fatos tornam necessário o desenvolvimento de pesquisas no âmbito das pilhas à combustível, objetivando, por exemplo, buscar novos compostos que possam desempenhar o papel da platina nesses equipamentos, a fim de substituí-la.

\section{OBJETIVO}

Sintetizar, caracterizar e investigar a atividade eletrocatalítica frente às reações de redução do oxigênio em meio básico de um composto de coordenação contendo centro metálico de níquel (II) coordenado a um ligante do tipo N,O-doador.

\section{METODOLOGIA EXPERIMENTAL}

\subsection{Síntese do ligante ácido 2-(imino-5-nitro-2-hidroxibenzil) -etanóico (NO2HBGi)}

O ligante ácido 2-(imino-5-nitro-2-hidroxibenzil) -etanóico foi sintetizado através da reação de condensação entre $5 \mathrm{mmol}$ de glicina e $5 \mathrm{mmol}$ de 5 -nitrosalicilaldeído, realizada num balão 


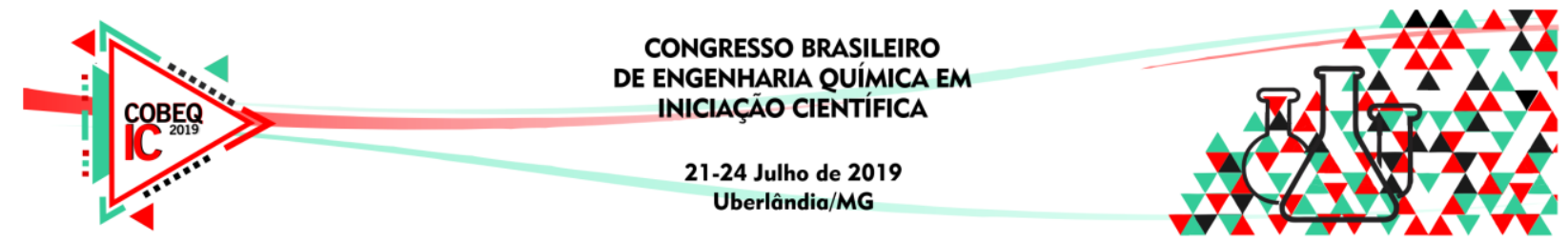

de fundo redondo contendo $100 \mathrm{~mL}$ de metanol e $5 \mathrm{mmol}$ de $\mathrm{KOH}$. O meio reacional foi mantido sob constante agitação por $2 \mathrm{~h}$. Em seguida, o $\mathrm{pH}$ do meio foi ajustado para 4.0, originando uma suspensão alaranjada.

Figura 1 - Esquema da síntese do ligante $\mathrm{NO}_{2} \mathrm{HBGi}$.<smiles>NCC(=O)Oc1ccc(O)c(C=NCC(=O)O)c1</smiles>

\subsection{Síntese do complexo $\mathrm{NiNO}_{2} \mathrm{HBGi}$}

A síntese do complexo foi realizada in situ, ou seja, foi sintetizado inicialmente o ligante (conforme descrito no item anterior) e, em seguida $30 \mathrm{~mL}$ de uma solução metanólica de $1,245 \mathrm{~g}$ ( $5 \mathrm{mmol})$ de acetato de níquel (II) tetrahidratado foram gotejados lentamente no meio reacional contendo o ligante $\mathrm{NO}_{2} \mathrm{HBGi}$, originando uma solução verde escura. A mistura foi mantida sob agitação magnética durante 2-3 horas e foi observada a formação de um precipitado verde, o qual foi filtrado. A solução mãe foi mantida em repouso à temperatura ambiente e após algumas semanas foi possível verificar a formação de cristais de coloração verde amarelado em forma de finíssimas agulhas, que foram considerados inadequados à análise por difração de raios $\mathrm{X}$ de monocristais.

Figura 2 - Esquema da síntese do complexo $\mathrm{NiNO}_{2} \mathrm{HBGi}$ (estrutura proposta).

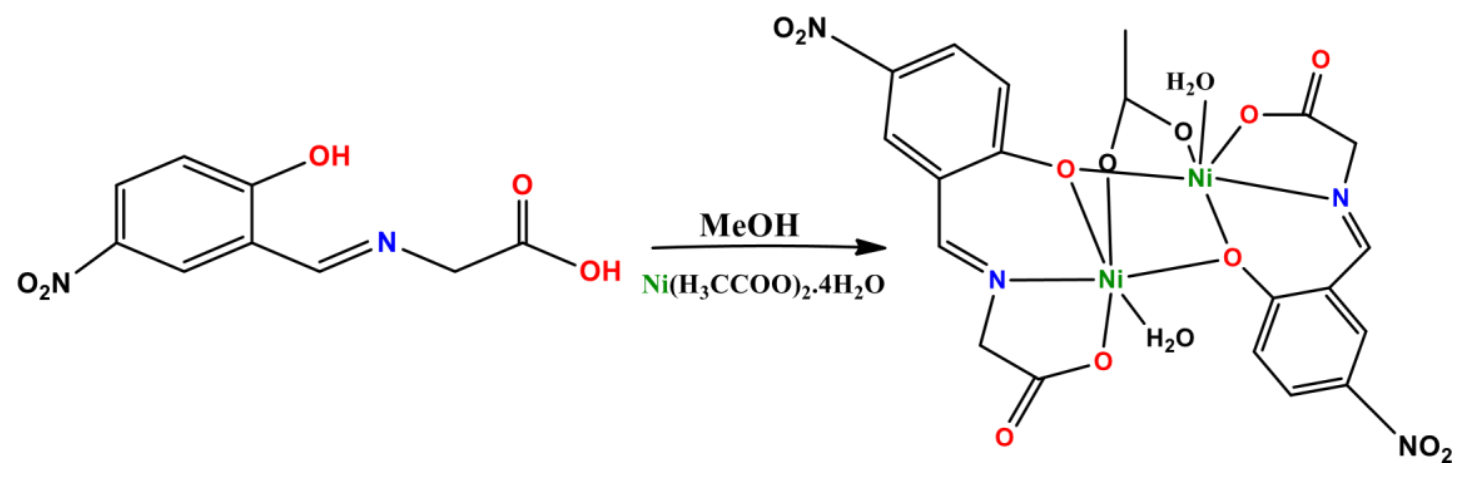

\section{RESULTADOS E DISCUSSÃO}

\subsection{Caracterização}

O ligante $\mathrm{NO}_{2} \mathrm{HBGi}$ e o complexo $\mathrm{NiNO}_{2} \mathrm{HBGi}$ foram caracterizados por técnicas espectroscópicas e eletroquímicas.

Espectroscopia no infravermelho: Os resultados obtidos através dos espectros no infravermelho do ligante $\mathrm{NO}_{2} \mathrm{HBGi}$ e do complexo $\mathrm{NiNO}_{2} \mathrm{HBGi}$ estão ilustrados a seguir:

Figura 3 - Espectro no Infravermelho dos compostos. 

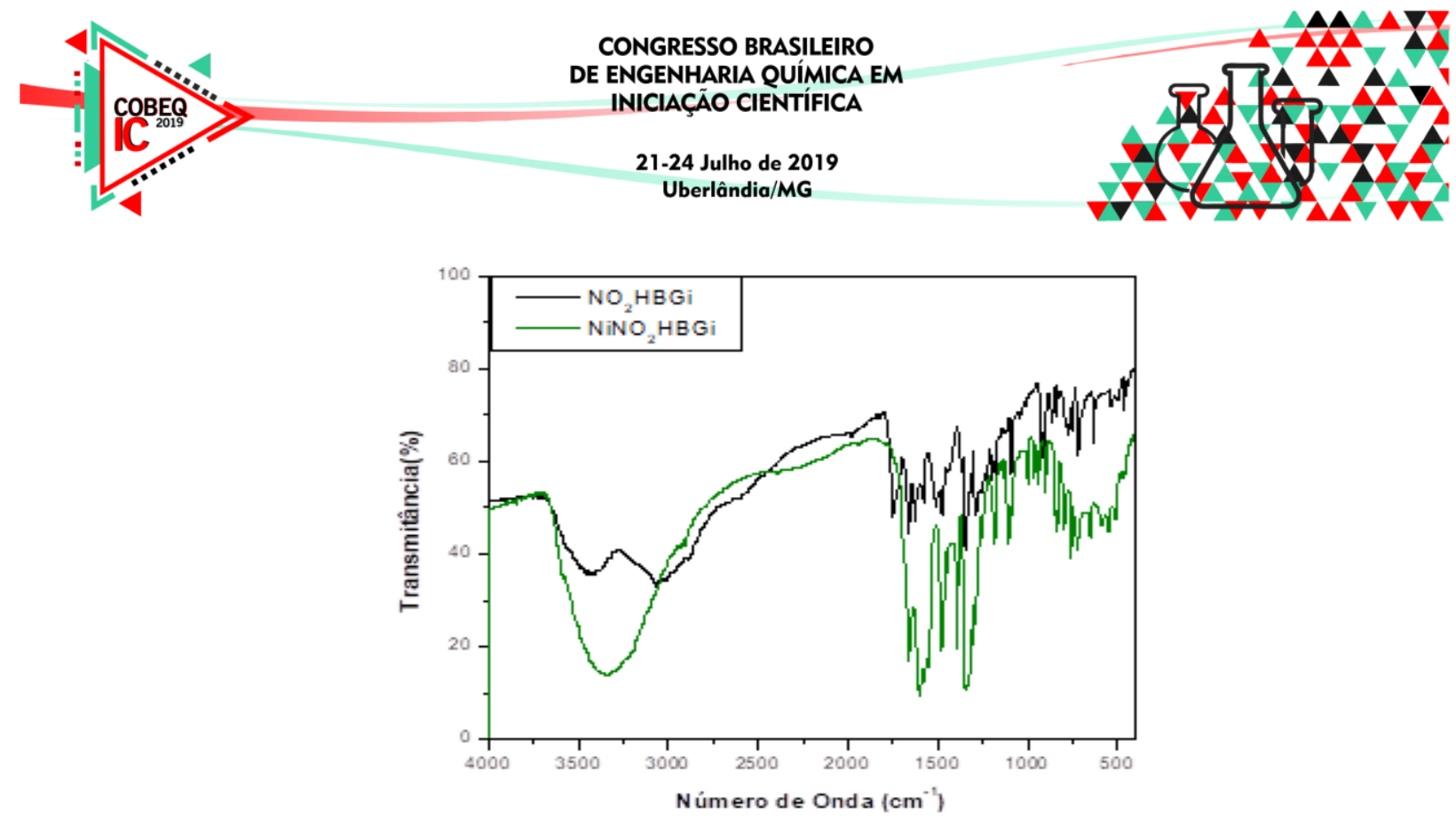

Tabela 1 - Atribuições dos valores das principais bandas observadas no espectro na região do infravermelho dos compostos

\begin{tabular}{|c|c|c|}
\hline Atribuições & $\mathrm{NO}_{2} \mathrm{HBGi}\left(\mathrm{cm}^{-1}\right)$ & $\mathrm{NiNO}_{2} \mathrm{HBGi}\left(\mathrm{cm}^{-1}\right)$ \\
\hline vO-H & 3434 & 3354 \\
\hline$v \mathrm{C}=\mathrm{N}$ & 1627 & 1602 \\
\hline vassi $\mathrm{COO}^{-}$ & - & 1574 \\
\hline$v_{\text {sim }} \mathrm{COO}^{-}$ & - & 1394 \\
\hline vC-O fenol & 1288 & 1301 \\
\hline v $\mathrm{C}=\mathrm{O}_{\text {ácido }}$ & 1665 & - \\
\hline VassiNO$_{2}$ & 1340 & 1342 \\
\hline $\mathrm{U}_{\operatorname{sim}} \mathrm{NO}_{2}$ & 1293 & 1251 \\
\hline vC-O fenol-ponte & - & 1661 \\
\hline v $\mathbf{C}=\mathbf{C}_{\text {arom. }}$. & $1520-1443$ & $1476-1446$ \\
\hline$v \mathbf{C}-\mathbf{N}$ & 1173 & 1109 \\
\hline vM-O & - & 547 \\
\hline vM-N & - & 487 \\
\hline
\end{tabular}

Analisando-se os valores das principais bandas do ligante e do complexo, é possível verificar um deslocamento das bandas para menores energias no espectro do complexo em relação ao ligante, o que sugere a ocorrência da coordenação do metal. 


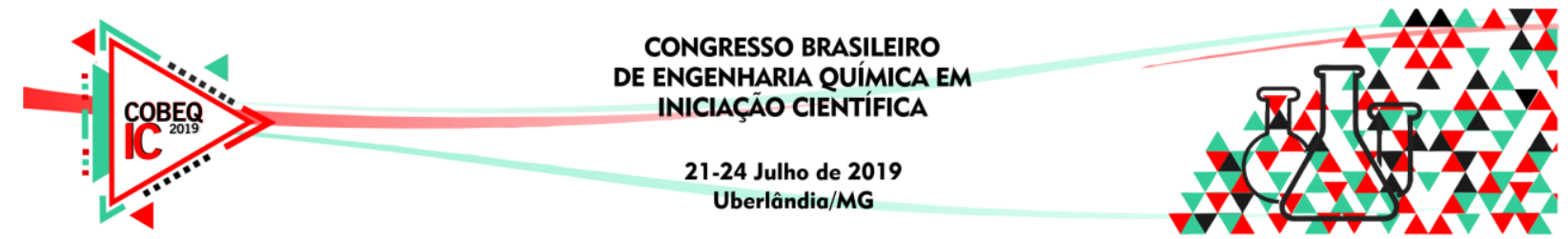

Se a diferença entre os valores das bandas do grupo acetato for em torno de $164 \mathrm{~cm}^{-1}$, pode indicar que esse grupo está ligado ao íon metálico como ponte. Para o complexo $\mathrm{NiNO}_{2} \mathrm{HBGi}$, essa diferença é de $180 \mathrm{~cm}^{-1}$, o que permite sugerir que o grupo acetato está ligado como ponte, reforçando a proposta de estrutura.

\section{UV-Vis/DRIFTs (Reflectância Difusa):}

Figura 4 - Deconvolução do espectro eletrônico na região do UV-Vis para o complexo.

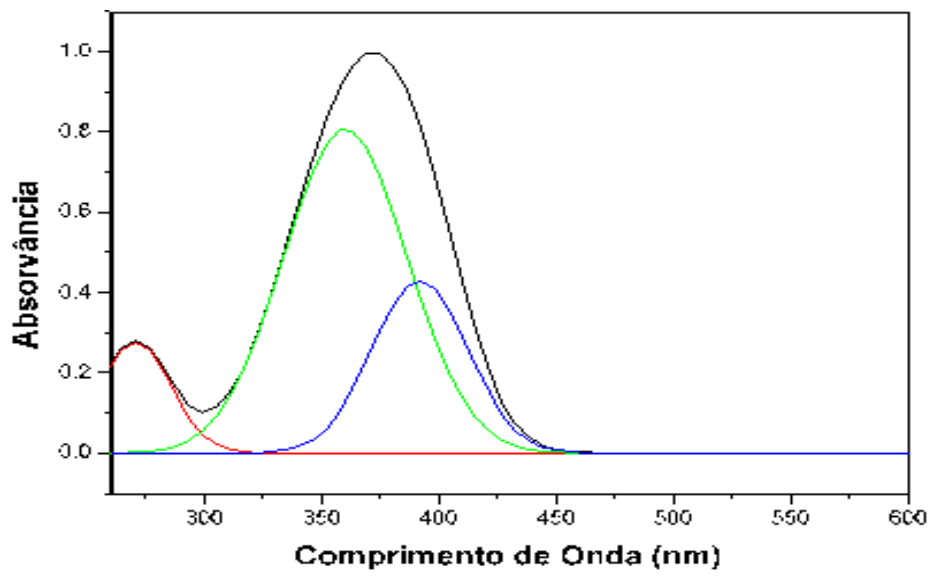

Figura 5 - Espectro eletrônico do complexo $\mathrm{NiNO}_{2} \mathrm{HBGi}$, obtido por reflectância difusa (em preto) na região de 200 a $800 \mathrm{~nm}$.

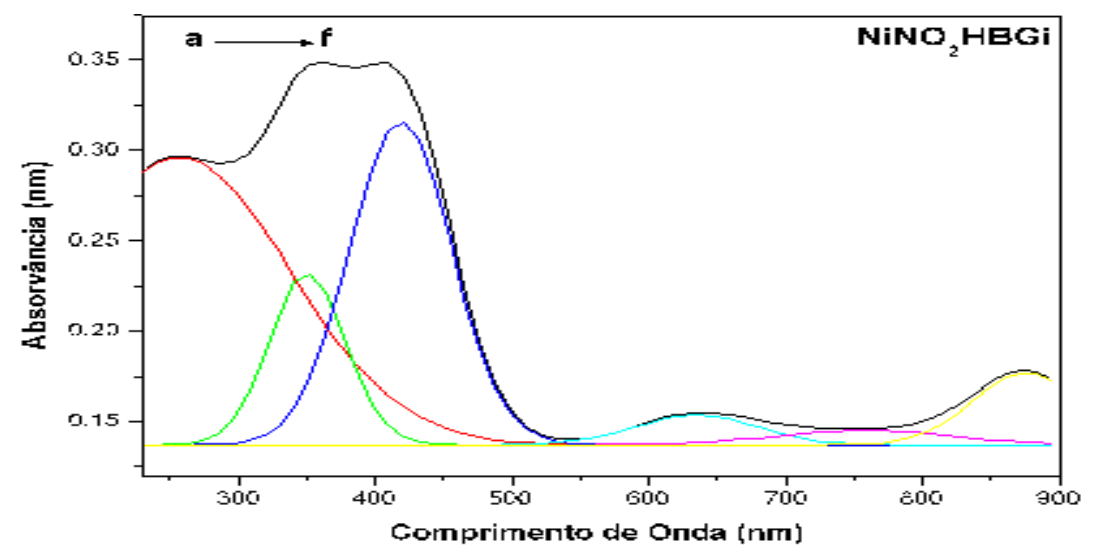

Tabela 2- Comparação e atribuições do espectro na região do UV-Vis com o DRIFTs do complexo

\begin{tabular}{|c|c|c|c|}
\hline DRIFT's & UV-Vis & $\begin{array}{c}\text { A bsortividade Molar } \\
\left(\mathrm{L} \cdot \mathrm{mol}^{-1} \cdot \mathrm{cm}^{-1}\right)\end{array}$ & Atribuição \\
\hline $\mathbf{8 7 8 n m}$ & $908 \mathrm{~nm}$ & 58,30 & $\mathrm{~d}-\mathrm{d}\left({ }^{3} \mathrm{~A}_{2 \mathrm{~g}} \rightarrow{ }^{3} \mathrm{~T}_{2 \mathrm{~g}}\right)$ \\
\hline $\mathbf{7 4 5 n m}$ & $736 \mathrm{~nm}$ & 39,42 & $\mathrm{~d}-\mathrm{d}\left({ }^{3} \mathrm{~A}_{2 \mathrm{~g}} \rightarrow{ }^{1} \mathrm{E}_{\mathrm{g}}\right)$ \\
\hline
\end{tabular}




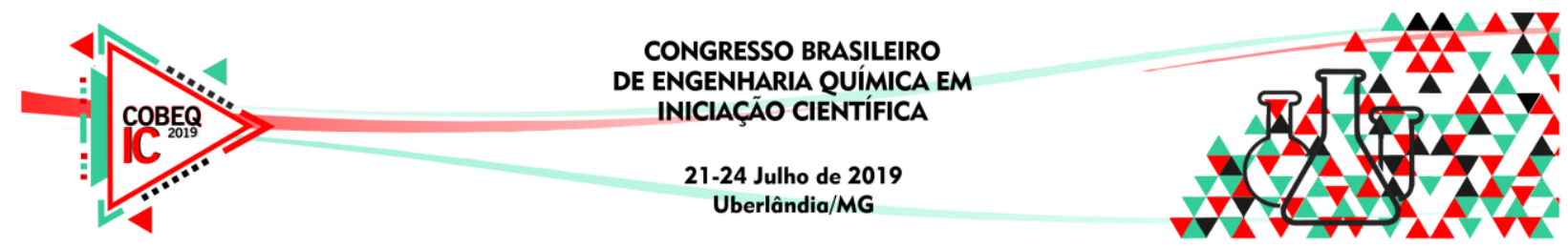

\begin{tabular}{|c|c|c|c|}
\hline $\mathbf{6 3 0 n m}$ & $615 \mathrm{~nm}$ & 48,93 & $\mathrm{~d}-\mathrm{d}\left({ }^{3} \mathrm{~A}_{2 \mathrm{~g}} \rightarrow{ }^{3} \mathrm{~T}_{1 \mathrm{~g}}(\mathrm{P})\right)$ \\
\hline $\mathbf{4 2 1 n m}$ & - & - & $\mathrm{TC}\left(\mathrm{NO}_{2}\right)$ \\
\hline $\mathbf{3 5 1 n m}$ & $382 \mathrm{~nm}$ & 64.815 & TCLM \\
\hline $\mathbf{2 5 5 n m}$ & $271 \mathrm{~nm}$ & 16.925 & TCIL \\
\hline
\end{tabular}

É possível verificar que os valores observados para o composto no estado sólido são similares aos encontrados em solução, sugerindo, assim, que o complexo não sofre alterações significativas na esfera de coordenação no estado sólido e em solução.

\section{Voltametria Cíclica:}

Figura 6 - Voltamograma cíclico do ligante $\mathrm{NO}_{2} \mathrm{HBGi}$ em $\mathrm{TBAPF}_{6} / \mathrm{DMSO}$ a $100 \mathrm{mV} / \mathrm{s}$ na

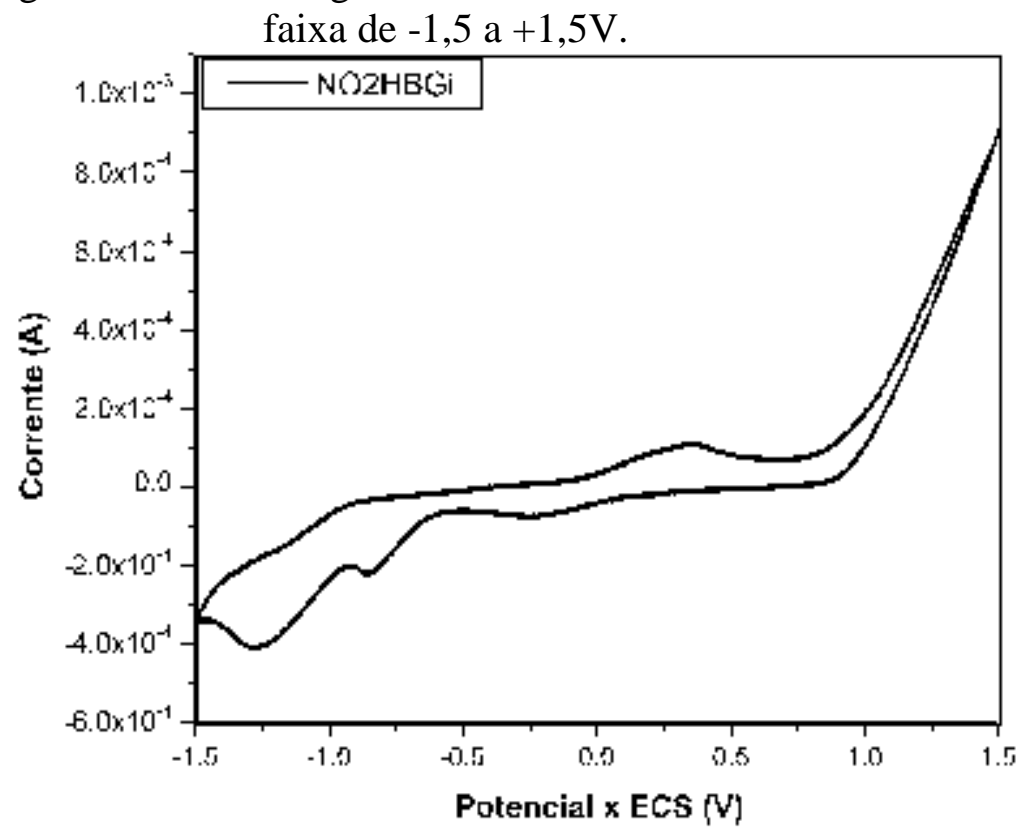

Figura 7 - Voltamogramas Cíclicos obtidos para o complexo $\mathrm{NiNO}_{2} \mathrm{HBGi}$, em atmosfera de $\mathrm{N}_{2}$, na faixa de potencial $-1,5 \mathrm{~V}$ à $1,5 \mathrm{~V}$, solução de TBAPF $6,05 \mathrm{~mol} \mathrm{~L}^{-1} \mathrm{em}$ DMSO espectroscópico com velocidade de varredura: 50, 75, 100 e $150 \mathrm{mV} \cdot \mathrm{s}^{-1}$.

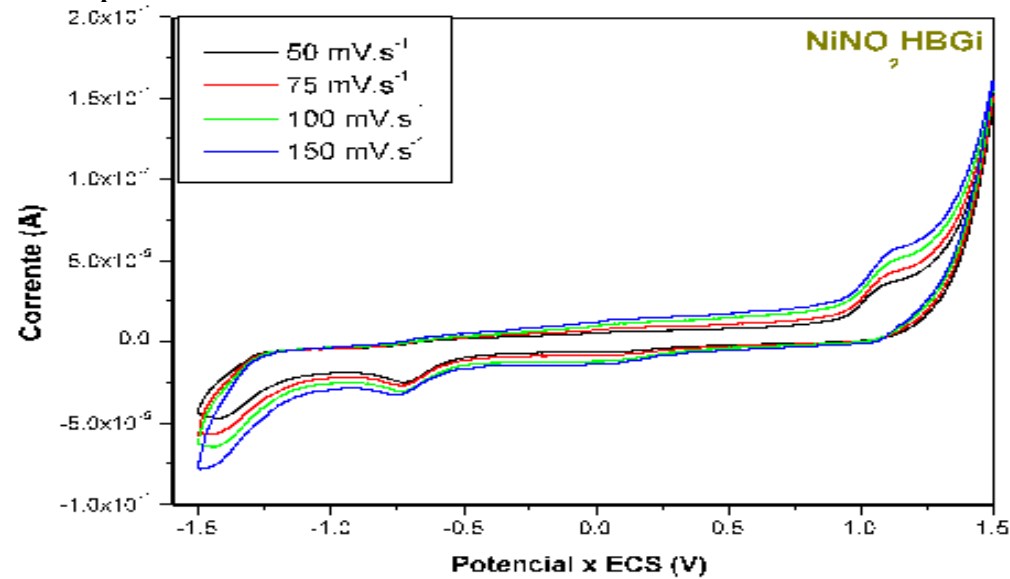




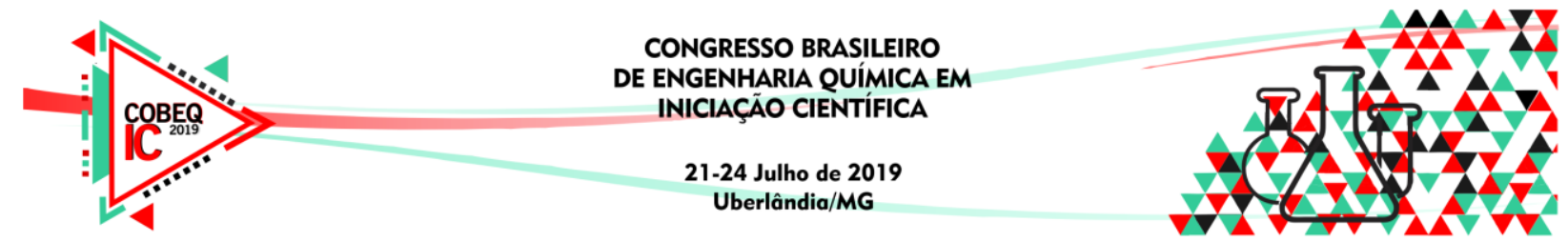

Comparando-se os voltamogramas de caracterização, é possível verificar no voltamograma do complexo o surgimento de um pico anódico em $+1,14 \mathrm{~V}$, relacionado a oxidação do centro metálico, ausente no ligante.

Atividade eletrocatalítica frente RRO em meio básico: No teste de atividade eletrocatalítica foi utilizada pasta de grafite modificada com o complexo estudado através da voltametria cíclica. Os experimentos foram realizados em atmosfera inerte e em oxigênio nas proporções de $30 \%(\mathrm{~m} / \mathrm{m})$ de complexo, na faixa de potencial $-1,0$ a $+1,0 \mathrm{~V}$ com velocidade de varredura $50 \mathrm{mV} . \mathrm{s}^{-1}$.

Figura 8 - Voltamograma cíclico da pasta $\mathrm{EPNiNO}_{2} \mathrm{HBGi}$ (eletrodo com pasta do complexo) e EPG (eletrodo com pasta de grafite) em $30 \%$ de complexo (m/m).

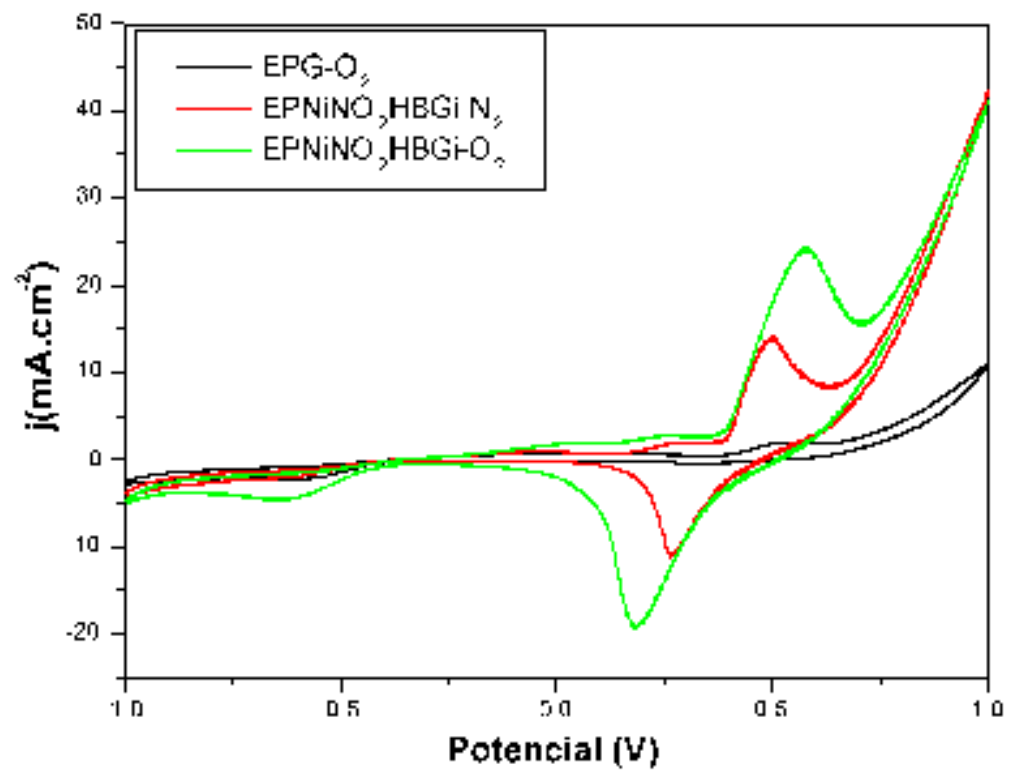

A pasta contendo o complexo apresentou um pico anódico em torno de $+0,58 \mathrm{~V}$ e um pico catódico em $-0,67 \mathrm{~V}$, o pico anódico está relacionado à oxidação do centro metálico referente ao par redox $\mathrm{Ni}^{2+} / \mathrm{Ni}^{3+}$ e o pico catódico a redução do oxigênio molecular.

\section{CONCLUSÃO}

Com base nos resultados obtidos, pode-se concluir que o composto em questão é promissor na eletrocatálise das reações de redução do oxigênio em meio básico.

\section{REFERÊNCIAS}

AMADO, Roberto Salgado et al. Pilhas a combustível de óxido sólido: materiais, componentes e configurações. Química Nova, v. 30, n. 1, p. 189-197, 2007.

HAN, Jing et al. Synthesis and characterization of three ionic pairs of Fe (II) and Co (II) complexes with tridentate salicylideneglycine. Journal of Coordination Chemistry, v. 62, n. 16, p. 2719-2727, 2009. 